International Journal of Pure and Applied Mathematics

Volume 92 No. $4 \quad 2014,549-563$

ISSN: 1311-8080 (printed version); ISSN: 1314-3395 (on-line version)

url: http://www.ijpam.eu

doi: http://dx.doi.org/10.12732/ijpam.v92i4.9

\title{
ASYMPTOTIC AND OSCILLATORY BEHAVIOUR OF CERTAIN NONLINEAR GENERALIZED $\alpha$-DIFFERENCE EQUATIONS
}

\author{
M. Maria Susai Manuel ${ }^{1}$, K. Srinivasan ${ }^{2}$, D.S. Dilip ${ }^{3}$, G. Dominic Babu ${ }^{4}$ \\ ${ }^{1}$ Department of Science and Humanities \\ R.M.D. Engineering College \\ Kavaraipettai, 601 206, Tamil Nadu, S. INDIA \\ ${ }^{2}$ Department of Science and Humanities \\ S.K.P. Institute of Technology \\ Tiruvannamalai, Tamil Nadu, S. INDIA \\ ${ }^{3,4}$ Department of Mathematics \\ Sacred Heart College \\ Tirupattur, 635 601, Vellore District, Tamil Nadu, S. INDIA
}

Abstract: In this paper, the authors discuss the asymptotic and oscillatory behavior of solutions of the nonlinear generalized $\alpha$-difference equation

$$
\Delta_{\alpha(\ell)}\left(p(k) \Delta_{\alpha(\ell)}(u(k)+q(k) u(k-\rho))\right)+t(k) f(u(k-\tau))=0, k \in[a, \infty),
$$

where the functions $p, q$ and $t$ are real numbers with $t(k) \geq 0$ and $\alpha, \ell, \rho, \tau$ are positive real. Further, $u f(u)>0$ for $u \neq 0, p(k)>0$ and

$$
R(k)=\sum_{r=0}^{\infty} \frac{1}{\alpha^{r} p(r \ell)}=\infty
$$

and $u(k)$ is defined for $k \geq-\max \{\rho, \tau\}$ for all $k \in[a, \infty)$ for some $a \in[0, \infty)$.

AMS Subject Classification: 39A12

Received: January 6, 2014

(C) 2014 Academic Publications, Ltd.

${ }^{\S}$ Correspondence author url: www.acadpubl.eu 
Key Words: generalized $\alpha$-difference equation, generalized $\alpha$-difference operator, oscillation and nonoscillation

\section{Introduction}

The basic theory of difference equations is based on the operator $\Delta$ defined as $\Delta u(k)=u(k+1)-u(k), k \in \mathbb{N}=\{0,1,2,3, \cdots\}$. Eventhough many authors ([1], [20]-[22]) have suggested the definition of $\Delta$ as

$$
\Delta u(k)=u(k+\ell)-u(k), k \in \mathbb{R}, \ell \in \mathbb{R}-\{0\},
$$

no significant progress took place on this line. But recently, E. Thandapani, M.M.S. Manuel, G.B.A.Xavier [5] considered the definition of $\Delta$ as given in (3) and developed the theory of difference equations in a different direction. For convenience, the operator $\Delta$ defined as (3) is labelled as $\Delta_{\ell}$ and by defining its inverse $\Delta_{\ell}^{-1}$, many interesting results and applications in number theory (see $[5],[11]-[19])$ were obtained. By extending the study related to the sequences of complex numbers and $\ell$ to be real, some new qualitative properties of the solutions like rotatory, expanding, shrinking, spiral and weblike were obtained for difference equation involving $\Delta_{\ell}$. The results obtained using $\Delta_{\ell}$ can be found in ([5]-[19]). Jerzy Popenda and B.Szmanda ([3],[4]) defined $\Delta$ as

$$
\Delta_{\alpha} u(k)=u(k+1)-\alpha u(k)
$$

and based on this definition, they studied the qualitative properties of a particular difference equation and no one else has handled this operator. In this paper, we have generalized the definition of $\Delta_{\alpha}$ given in (4) and defined and denoted it as

$$
\Delta_{\alpha(\ell)} u(k)=u(k+\ell)-\alpha u(k) .
$$

where $\alpha>1$ and $\ell \in[0, \infty)$ and by defining its inverse, several interesting results on number theory were obtained.

In [2], Aleksandra Sternal and Blazej Szmanda obtained sufficient conditions for the asymptotic and oscillatory behaviour of the difference equations are of the form $\Delta_{\alpha(\ell)}\left(p(k) \Delta_{\alpha(\ell)}(u(k)+q(k) u(k-\rho))\right)+t(k) f(u(k-\tau))=0, k \in[a, \infty)$. In this paper the theory is extended from $\Delta$ to $\Delta_{\alpha(\ell)}$ for all real $k \in[a, \infty)$ and we discuss the asymptotic and oscillatory behavior of solutions of generalized $\alpha$-difference equation (1).

Throughout this paper, we make use the following assumptions:

(a) $\mathbb{N}=\{0,1,2,3, \ldots\}, \mathbb{N}(a)=\{a, a+1, a+2, \ldots\}$, 
(b) $\mathbb{N}_{\ell}(a)=\{a, a+\ell, a+2 \ell, \ldots\}$.

(c) $\lceil x\rceil$ and $[x]$ denotes the upper integer and the integer part of $x$ respectively.

(d) $j=k-k_{i}-\left[\frac{k-k_{i}}{\ell}\right] \ell, k_{i} \in[0, \infty)$.

(e) $f(u)$ is bounded away from zero, if $u$ is bounded away from zero.

(f) $\sum_{r=0}^{\infty} t(r \ell)=\infty$.

(g) $z(k)=u(k)+q(k) u(k-\rho)$.

\section{Preliminaries}

In this section, we present some preliminaries which will be useful for future discussion.

Definition 2.1. [14] The inverse of the Generalized $\alpha$-difference operator denoted by $\Delta_{\alpha(\ell)}^{-1}$ on $u(k)$ is defined as follows. If $\Delta_{\alpha(\ell)} v(k)=u(k)$, then

$$
\Delta_{\alpha(\ell)}^{-1} u(k)=v(k)-\alpha^{\left[\frac{k}{\ell}\right]} v(j)
$$

where $k \in \mathbb{N}_{\ell}(j), j=k-\left[\frac{k}{\ell}\right] \ell$.

Lemma 2.2. [5] If the real valued function $u(k)$ is defined for all $k \in[a, \infty)$ and $\alpha>1$, then

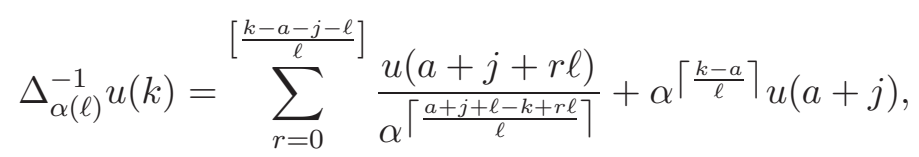

for all $k \in \mathbb{N}_{\ell}(j), j=k-a-\left[\frac{k-a}{\ell}\right] \ell$.

Definition 2.3. [14] The solution $u(k)$ of (1) is called oscillatory if for any $k_{1} \in[a, \infty)$ there exists a $k_{2} \in \mathbb{N}_{\ell}\left(k_{1}\right)$ such that $u\left(k_{2}\right) u\left(k_{2}+\ell\right) \leq 0$. The difference equation itself is called oscillatory if all its solutions are oscillatory. If the solution $u(k)$ is not oscillatory, then it is said to be nonoscillatory (i.e. $u(k) u(k+\ell)>0$ for all $\left.k \in\left[k_{1}, \infty\right)\right)$. 


\section{Main Results}

In this section we establish conditions for the asymptotic and oscillatory behaviour of solutions of equation (1).

Lemma 3.1. Assume that there exists a constant $P_{1}<0$ such that $P_{1} \leq q(k) \leq 0$.

i) If $u(k)$ is an eventually positive solution of (1), then $z(k)$ and $p(k) \Delta_{\alpha(\ell)} z(k)$ are eventually monotonic and either

$$
\lim _{k \rightarrow \infty} z(k)=\lim _{k \rightarrow \infty} p(k) \Delta_{\alpha(\ell)} z(k)=-\infty
$$

or

$$
\lim _{k \rightarrow \infty} z(k)=\lim _{k \rightarrow \infty} p(k) \Delta_{\alpha(\ell)} z(k)=0, \Delta_{\alpha(\ell)} z(k)>0 \text { and } z(k)<0 .
$$

In addition, if $P_{1} \geq-1$, then (9) holds and $u(k)$ is bounded.

ii) If $u(k)$ is an eventually negative solution of (1), then the sequences $z(k)$ and $p(k) \Delta_{\alpha(\ell)} z(k)$ are monotonic and either

$$
\lim _{k \rightarrow \infty} z(k)=\lim _{k \rightarrow \infty} p(k) \Delta_{\alpha(\ell)} z(k)=\infty
$$

or

$$
\lim _{k \rightarrow \infty} z(k)=\lim _{k \rightarrow \infty} p(k) \Delta_{\alpha(\ell)} z(k)=0, \Delta_{\alpha(\ell)} z(k)<0 \text { and } z(k)>0
$$

In addition, if $P_{1} \geq-1$, then (11) holds and $u(k)$ is bounded.

Proof. Let $u(k)$ be an eventually positive solution of (1). Then, from (1) there exists a positive integer $k_{1}$ such that

$$
\Delta_{\alpha(\ell)}\left(p(k) \Delta_{\alpha(\ell)} z(k)\right)=-t(k) f(u(k-\tau)) \leq 0 \text { for } k \geq k_{1},
$$

that is, $p(k) \Delta_{\alpha(\ell)} z(k)$ is nonincreasing, which implies that $\Delta_{\alpha(\ell)} z(k)$ is eventually of constant sign and in consequence $z(k)$ is monotonic.

First, let there exists $k_{2} \geq k_{1}$ such that $\Delta_{\alpha(\ell)} z\left(k_{2}\right) \leq 0$, then, since $t(k) \neq 0$ eventually, there exists $k_{3}>k_{2}$ such that

$$
p(k) \Delta_{\alpha(\ell)} z(k) \leq \alpha^{\left\lceil\frac{k-k_{3}}{\ell}\right\rceil} p\left(k_{3}\right) \Delta_{\alpha(\ell)} z\left(k_{3}\right)=c<0
$$

for $k \geq k_{3}$. 
From Definition 2.1 and Lemma 2.2, we have

$$
\begin{aligned}
& z(k) \leq \alpha^{\left\lceil\frac{k-k_{3}}{\ell}\right\rceil} z\left(k_{3}\right)+c \sum_{r=0}^{\frac{k-k_{3}-\ell-j}{\ell}} \frac{1}{\alpha^{\left\lceil\frac{k_{3}+j+\ell-k+r \ell}{\ell}\right\rceil} p\left(k_{3}+j+\ell+r \ell\right)} \\
& \rightarrow-\infty \text { as } k \rightarrow \infty
\end{aligned}
$$

and hence $z(k) \rightarrow-\infty$ as $k \rightarrow \infty$. Since $p(k) \Delta_{\alpha(\ell)} z(k)$ is nonincreasing, we have

$$
p(k) \Delta_{\alpha(\ell)} z(k) \rightarrow L \geq-\infty .
$$

If $-\infty<L<0$, applying Definition 2.1 and Lemma 2.2 to (12), we get $p(k+$ $\ell) \Delta_{\alpha(\ell)} z(k+\ell)=\alpha^{\left\lceil\frac{k-k_{3}}{\ell}\right\rceil} p\left(k_{3}+j\right) \Delta_{\alpha(\ell)} z\left(k_{3}+j\right)-$

$$
\sum_{r=0}^{\frac{k-k_{3}-j}{\ell}} \frac{t\left(k_{3}+j+r \ell\right) f\left(u\left(k_{3}+j+r \ell-\tau\right)\right)}{\alpha^{\left\lceil\frac{k_{3}+j-k+r \ell}{\ell}\right\rceil}}
$$

and then let $k \rightarrow \infty$ to obtain $\sum_{r=0}^{\infty} \frac{t\left(k_{3}+j+r \ell\right) f\left(u\left(k_{3}+j+r \ell-\tau\right)\right)}{\alpha \frac{\left[\frac{k_{3}+j-k+r \ell}{\ell}\right\rceil}{\ell}}$

$$
=\alpha^{\left\lceil\frac{k-k_{3}}{\ell}\right\rceil} p\left(k_{3}+j\right) \Delta_{\alpha(\ell)} z\left(k_{3}+j\right)-L<\infty .
$$

The last inequality together with (e) and (f) implies $\liminf _{k \rightarrow \infty} u(k)=0$. Since $z(k)$ is eventually negative, we can choose $k_{4}>k_{3}$ such that $p(k) \Delta_{\alpha(\ell)} z(k)<\frac{L}{2}$ for $k \geq k_{4}$ and $z\left(k_{4}\right)<0$. Applying Definition 2.1 and Lemma 2.2 to the above inequality we obtain

$$
\begin{aligned}
z(k) & <\alpha^{\left\lceil\frac{k-k_{3}}{\ell}\right\rceil} z\left(k_{4}\right)+\frac{L}{2} \sum_{r=0}^{\frac{k-k_{4}-\ell-j}{\ell}} \frac{1}{\alpha^{\left\lceil\frac{k_{4}+j+\ell-k+r \ell}{\ell}\right]} p\left(k_{4}+j+\ell+r \ell\right)} \\
& <\frac{L}{2} \sum_{r=0}^{\frac{k-k_{4}-\ell-j}{\ell}} \frac{1}{\alpha^{\left\lceil\frac{k_{4}+j+\ell-k+r \ell}{\ell}\right\rceil} p\left(k_{4}+j+\ell+r \ell\right)}, \text { for } k>k_{4} .
\end{aligned}
$$

By the assumptions, we have $P_{1} u(k-\rho) \leq q(k) u(k-\rho)<z(k)$

$$
<\frac{L}{2} \sum_{r=0}^{\frac{k-k_{4}-\ell-j}{\ell}} \frac{1}{\alpha^{\left\lceil\frac{k_{4}+j+\ell-k+r \ell}{\ell}\right]} p\left(k_{4}+j+\ell+r \ell\right)}, k>k_{4}
$$


and

$$
u(k-\rho)>\frac{L}{2 P_{1}} \sum_{r=0}^{\frac{k-k_{4}-\ell-j}{\ell}} \frac{1}{\alpha^{\left\lceil\frac{k_{4}+j+\ell-k+r \ell}{\ell}\right\rceil} p\left(k_{4}+j+\ell+r \ell\right)} \rightarrow \infty,
$$

as $k \rightarrow \infty$ which contradicts $\liminf _{k \rightarrow \infty} u(k)=0$. Thus, $\lim _{k \rightarrow \infty} p(k) \Delta_{\alpha(\ell)} z(k)=-\infty$.

Now, if $\Delta_{\alpha(\ell)} z(k)>0$ for $k \geq k_{1}$, then $p(k) \Delta_{\alpha(\ell)} z(k) \rightarrow L_{1} \geq 0$ as $n \rightarrow \infty$. As before, summing (12) from $k \geq k_{1}$ to $m$ and letting $m \rightarrow \infty$ we obtain

$$
p(k) \Delta_{\alpha(\ell)} z(k)=L_{1}+\sum_{r=0}^{\infty} \frac{t(k+r \ell) f(u(k+r \ell-\tau))}{\alpha^{r}}
$$

which again implies that $\liminf _{n \rightarrow \infty} u(k)=0$.

Suppose that $L_{1}>0$. Then we have, $p(k) \Delta_{\alpha(\ell)} z(k) \geq L_{1}>0$ and a summation shows that $z(k) \rightarrow \infty$ as $n \rightarrow \infty$. Since $u(k) \geq z(k)$, which leads to $u(k) \rightarrow \infty$ as $k \rightarrow \infty$, a contradiction. Therefore, $L_{1}=0$. Furthermore, if there exists $k_{2} \geq k_{1}$ such that $z\left(k_{2}\right) \geq 0$, then $\Delta_{\alpha(\ell)} z(k)>0$ implies that $z(k) \geq z\left(k_{3}\right)>0$ for all $k \geq k_{3}$ and some $k_{3}>k_{2}$, which again contradicts $\liminf u(k)=0$. Therefore, we have $z(k)<0$ for $k \geq k_{1}$. Thus $z(k) \rightarrow L_{2} \leq 0$. If $\vec{L}_{2}<0$, then

$$
P_{1} u(k-\rho) \leq u(k)+q(k) u(k-\rho)=z(k) \leq L_{2}<0 \text { for } k \geq k_{1}
$$

and

$$
u(k-\rho)>\frac{L_{2}}{P_{1}}>0, k \geq k_{1},
$$

which contradicts $\liminf _{n \rightarrow \infty} u(k)=0$. Therefore $L_{2}=0$.

Now we assume that $P_{1} \leq-1$. Suppose that (9) does not hold. Then (8) holds, so $z(k)<0$ for all large $k$ and we have

$$
u(k)<-q(k) u(k-\rho) \leq-P_{1} u(k-\rho) \leq u(k-\rho)
$$

for all large $k$. But the last inequality implies that $u(k)$ is bounded which contradicts (8). Therefore, (9) holds and $u(k)$ is a bounded solution of (1). The proof of (ii) is similar to that of (i) and hence will be omitted.

Theorem 3.2. If there exists a constant $P_{1}$ such that

$$
-1<P_{1} \leq q(k) \leq 0,
$$

then, every nonoscillatory solution $u(k)$ of (1) tends to 0 as $k \rightarrow \infty$. 
Proof. If $u(k)$ is an eventually positive solution of (1), then by part i) of Lemma 3.1, we see that $u(k)$ is a bounded solution of (1).

Now, suppose that $\lim \sup u(k)=a>0$. Then, there exists a subsequence

$$
\lim _{k \rightarrow \infty}
$$

of $u(k)$, say $u\left(k_{i}\right)$ such that $u\left(k_{i}\right) \rightarrow a$ as $i \rightarrow \infty$. Then, for all large $i$, we have

$$
0>z\left(k_{i}\right) \geq u\left(k_{i}\right)+P_{1} u\left(k_{i}-\rho\right) \text { so } u\left(k_{i}-\rho\right)>-\frac{u\left(k_{i}\right)}{P_{1}} .
$$

But this implies that $\lim _{i \rightarrow \infty} u\left(k_{i}-\rho\right) \geq-\frac{a}{P_{1}}>a$, contradicting the choice of $a$.

Therefore, $u(k) \rightarrow 0$ as $k \rightarrow \infty$. The proof when $u(k)$ is eventually negative is similar.

Example 3.3. For the generalized $\alpha$-difference equation

$$
\begin{gathered}
\Delta_{\alpha(\ell)}\left(\frac{1}{k} \Delta_{\alpha(\ell)}\left(\frac{1}{k^{2}}-\frac{1}{k(k-2 \ell)^{2}}\right)\right)=\frac{1}{\left((k+2 \ell)^{2}\right)_{\ell}^{(5)}(k+\ell)_{\ell}^{(2)}} \\
\left(k\left(k^{2}-k-2 \ell\right)\left(k^{2}-\ell^{2}\right)^{2}(k-2 \ell)^{2}-\alpha\left[(k-\ell)^{2}-(k+\ell)\right] k^{2}(2 k+\ell)\left(k^{2}-4 \ell^{2}\right)^{2}\right. \\
\left.+\alpha^{2}\left[(k-2 \ell)^{2}-k\right](k+2 \ell)^{2}\left(k^{2}-\ell^{2}\right)^{2}(k+\ell)\right)
\end{gathered}
$$

where $q(k)=\frac{-1}{k}, p(k)=\frac{1}{k}$ and the conditions of Theorem 3.2 hold and ultimately every nonoscillatory solution $u(k)$ of (15) tends to zero as $k \rightarrow \infty$. Infact $u(k)=\frac{1}{k^{2}}$ is one such solution.

The following theorem is an easy consequence of Theorem 3.2.

Theorem 3.4. If $-1 \leq q(k) \leq 0$, then every unbounded solution of (1) is oscillatory.

The following theorem shows that, if $q(k)$ is bounded with upper bound less than -1 , then all bounded nonoscillatory solutions of (1) tend to zero as $k \rightarrow \infty$.

Theorem 3.5. If there exist constants $P_{1}$ and $P_{2}$ such that

$$
P_{1} \leq q(k) \leq P_{2}<-1
$$

then every bounded solution $u(k)$ of (1) is either oscillatory or satisfies $u(k) \rightarrow 0$ as $k \rightarrow 0$. 
Proof. Assume that (1) has a bounded nonoscillatory solution $u(k)$ and let $u(k)$ be eventually positive. By part (i) of Lemma 3.1 either (8) or (9) holds. Clearly (8) cannot hold in view of (16) and the fact that $u(k)$ is bounded. From (9) we have $z(k)<0$ and $z(k) \rightarrow 0$ as $k \rightarrow \infty$. Therefore, for any number $\epsilon>0$ there exists $k_{1}$ so that for $k \geq k_{1}$ we have

$$
-\epsilon<z(k) \leq u(k)+P_{2} u(k-\rho)
$$

or

$$
u(k-\rho)<-\frac{u(k)+\epsilon}{P_{2}} .
$$

So

$$
u(k)<-\frac{1}{P_{2}} u(k+\rho)-\frac{1}{P_{2}} \epsilon
$$

and further

$$
u(k+\rho)<-\frac{1}{P_{2}} u(k+2 \rho)-\frac{1}{P_{2}} \epsilon .
$$

From (17) and (18) we get

$$
u(k)<\left(-\frac{1}{P_{2}}\right)^{2} u(k+2 \rho)+\left(-\frac{1}{P_{2}}\right)^{2} \epsilon+\left(-\frac{1}{P_{2}}\right)^{2} \epsilon .
$$

After $m$ iterations, we obtain

$$
u(k)<\left(-\frac{1}{P_{2}}\right)^{m} u(k+m \rho)+\epsilon \sum_{i=1}^{m}\left(-\frac{1}{P_{2}}\right)^{i} .
$$

Let $\lambda=1+\frac{1}{P_{2}}>0$ and $u(k)<M$. Now, choose $m$ large enough so that $\left(-\frac{1}{P_{2}}\right)^{m}<\frac{\epsilon}{\lambda M}$. Thus, for every $\epsilon>0$ there exists $k_{2} \geq k_{1}$ such that for $k \geq k_{2}$ we have

$$
u(k)<\frac{\epsilon}{\lambda}+\epsilon\left(-\frac{1}{P_{2}}\right) \frac{1-\left(-\frac{1}{P_{2}}\right)^{m}}{1+\frac{1}{P_{2}}}<2 \frac{\epsilon}{\lambda} .
$$

That is, $u(k) \rightarrow 0$ as $k \rightarrow \infty$.

The proof when $u(k)$ is eventually negative is similar.

Example 3.6. For the generalized $\alpha$-difference equation

$$
\Delta_{\alpha(\ell)}\left(\frac{1}{k} \Delta_{\alpha(\ell)}\left(\frac{1}{k^{2}}+\frac{(1-2 k)}{k(k-2 \ell)^{2}}\right)\right)=\frac{1}{\left((k+2 \ell)^{2}\right)_{\ell}^{(5)}(k+\ell)_{\ell}^{(2)}}
$$




$$
\begin{array}{r}
\left((k+\ell)^{2} k\left(k^{2}+(1-2(k+2 \ell))\right)(k+2 \ell)+\alpha \ell\left(k^{2}+6 k \ell+\ell^{2}-k-\ell\right)\right. \\
\left.(k+2 \ell)^{2} k^{2}(k-2 \ell)^{2}+\alpha^{2}\left[(k-2 \ell)^{2}+(1-2 k) k\right](k+2 \ell)^{2}(k+\ell)^{3}(k-\ell)^{2}\right)
\end{array}
$$

where $q(k)=\frac{1-2 k}{k}, p(k)=\frac{1}{k}$ all the conditions of Theorem 3.5 hold and ultimately every every bounded solution $u(k)$ of (19) satisfies $u(k) \rightarrow 0$ as $k \rightarrow \infty$. Infact, $u(k)=\frac{1}{k^{2}}$ is one such solution.

Theorem 3.7. If $q(k)$ is eventually nonnegative, then any solution $u(k)$ of (1) is either oscillatory or satisfies $\liminf _{k \rightarrow \infty}|u(k)|=0$.

Proof. Let $u(k)$ be a nonoscillatory solution of (1) and assume that $u(k)$ is eventually positive. Then, as before, (12) implies that $p(k) \Delta_{\alpha(\ell)} z(k)$ is nonincreasing and also $z(k)>0$ eventually, say for $k \geq k_{1}$. It is easy to see that $\Delta_{\alpha(\ell)} z(k)>0$ for $k \geq k_{1}$. Indeed, if there exists $k_{2} \geq k_{1}$ such that $\Delta_{\alpha(\ell)} z\left(k_{2}\right) \leq$ 0 , then there exists $k_{3} \geq k_{2}$ such that $p(k) \Delta_{\alpha(\ell)} z(k) \leq p\left(k_{3}\right) \Delta_{\alpha(\ell)} z\left(k_{3}\right)=c<0$, since $p(k) \Delta_{\alpha(\ell)} z(k)$ is nonincreasing and $t(k) \equiv 0$ eventually. By (2), we get

$$
\begin{aligned}
z(k) \leq \alpha^{\left\lceil\frac{k-k_{3}}{\ell}\right\rceil} z\left(k_{3}\right)+c & \sum_{r=0}^{\frac{k-k_{3}-\ell-j}{\ell}} \frac{1}{\alpha{ }^{\left[\frac{k_{3}+j+\ell-k+r \ell}{\ell}\right]} p\left(k_{3}+j+\ell+r \ell\right)} \\
\rightarrow-\infty & \text { as } k \rightarrow \infty,
\end{aligned}
$$

which contradicts that $z(k)>0$ for $k \geq k_{1}$.

Therefore, $p(k) \Delta_{\alpha(\ell)} z(k) \rightarrow L \geq 0$ as $k \rightarrow \infty$. Summing (12) from $k$ to $m>n$ with $k$ sufficiently large and then letting $m \rightarrow \infty$ we obtain

$$
\sum_{r=0}^{\infty} \frac{t(k+r \ell) f(u(k+r \ell-\tau))}{\alpha^{\left\lceil\frac{k+r \ell}{\ell}\right\rceil}}=p(k) \Delta_{\alpha(\ell)} z(k)-L<\infty
$$

which, by (e) and (f), implies that $\liminf _{k \rightarrow \infty} u(k)=0$. The proof for $u(k)$ eventually negative is similar.

Example 3.8. For the generalized $\alpha$-difference equation

$$
\begin{gathered}
\Delta_{\alpha(\ell)}\left(\frac{1}{k} \Delta_{\alpha(\ell)}\left(\frac{1}{k^{2}}+\frac{1}{k(k-2 \ell)^{2}}\right)\right)=\frac{1}{\left((k+2 \ell)^{2}\right)_{\ell}^{(5)}(k+\ell)_{\ell}^{(2)}} \\
\left(k\left(k^{2}+k+2 \ell\right)\left(k^{2}-\ell^{2}\right)^{2}(k-2 \ell)^{2}-\alpha\left[(k-\ell)^{2}+(k+\ell)\right] k^{2}(2 k+\ell)\left(k^{2}-4 \ell^{2}\right)^{2}\right. \\
\left.+\alpha^{2}\left[(k-2 \ell)^{2}-k\right](k+2 \ell)^{2}\left(k^{2}-\ell^{2}\right)^{2}(k+\ell)\right)
\end{gathered}
$$


where $q(k)=\frac{1}{k}, p(k)=\frac{1}{k}$ and the conditions of Theorem 3.7 hold and ultimately every nonoscillatory solution $u(k)$ of $(21)$ tends to zero as $k \rightarrow \infty$. Infact, $u(k)=\frac{1}{k^{2}}$ is one such solution.

Theorem 3.9. If $0 \leq q(k) \leq q, t(k) \geq t>0$ and there exists a constant $A>0$ such that $|f(u)| \geq A|u|$ for all $u$, then all solutions of (1) are oscillatory.

Proof. Arguing as in the proof of Theorem 3.7 for an eventually positive solution $u(k)$ of (1) we get the equality (20). Further, the assumption, (20) gives

$$
A q \sum_{r=0}^{\infty} \frac{u(k+r \ell-\tau)}{\alpha^{\left\lceil\frac{k+r \ell}{\ell}\right\rceil}} \leq p(k) \Delta_{\alpha(\ell)} z(k)-L<\infty,
$$

which implies that $u(k) \rightarrow 0$ as $k \rightarrow \infty$ and so $z(k) \rightarrow 0$ as $k \rightarrow \infty$, which contradicts the fact that $z(k)>0$ and $\Delta_{\alpha(\ell)} z(k)>0$. The proof is now complete.

Example 3.10. For the generalized $\alpha$-difference equation

$$
\begin{gathered}
\Delta_{\alpha(\ell)}\left(\frac{1}{k} \Delta_{\alpha(\ell)}\left((-\alpha)^{\left\lceil\frac{k}{\ell}\right\rceil}+\frac{1}{k}(-\alpha)^{\left\lceil\frac{k-2 \ell}{\ell}\right\rceil}\right)\right)=\frac{(-\alpha)^{\left\lceil\frac{k+2 \ell}{\ell}\right\rceil}}{(k+\ell) k} \\
\left(\left(1+\frac{\alpha^{2}}{(k+2 \ell)}\right) k+\left(1+\frac{\alpha^{2}}{(k+\ell)}\right)(2 k+\ell)+\left(1+\frac{\alpha^{2}}{k}\right)(k+\ell)\right)
\end{gathered}
$$

where $q(k)=\frac{1}{k}, p(k)=\frac{1}{k}$, all the conditions of Theorem 3.9 hold and hence all solutions $u(k)$ of $(22)$ are oscillatory. Infact $u(k)=(-\alpha)^{\left\lceil\frac{k}{\ell}\right\rceil}$ is one such solution.

Theorem 3.11. Let $q(k) \geq 0$. Then every nonoscillatory solution $u(k)$ of (1) satisfies the following:

(i) $|u(k)| \leq b R(k)$ for some constant $b>0$ and all large $k$,

(ii) if $\left(\frac{R(k)}{t(k)}\right)$ is bounded, then $u(k)$ is bounded,

(iii) if $\frac{R(k)}{t(k)} \rightarrow 0$ as $k \rightarrow \infty$, then $u(k) \rightarrow 0$ as $k \rightarrow \infty$, where $R(k)=$ $\sum_{r=0}^{\left[\frac{k-\ell}{\ell}\right]} \frac{1}{\alpha^{r} p(r \ell)}$. 
Proof. Let $u(k)$ be an eventually positive solution of (1). As before, from (1) we have $\Delta_{\alpha(\ell)}\left(p(k) \Delta_{\alpha(\ell)} z(k)\right) \leq 0$ for $k \geq k_{1}$, so summing twice we get

$$
\begin{gathered}
z(k) \leq \alpha^{\left\lceil\frac{k-k_{3}}{\ell}\right\rceil} z\left(k_{1}\right)+p\left(k_{1}\right) \Delta_{\alpha(\ell)} z\left(k_{1}\right) \times \\
\times \sum_{r=0}^{\frac{k-k_{3}-\ell-j}{\ell}} \frac{1}{\alpha^{\left\lceil\frac{k_{3}+j+\ell-k+r \ell}{\ell}\right]} p\left(k_{3}+j+\ell+r \ell\right)}, k>k_{1} .
\end{gathered}
$$

By condition (2), we conclude that there is a constant $b>0$ such that $z(k) \leq$ $b R(k), k \geq k_{2}>k_{1}$. Clearly $u(k) \leq b R(k)$, so (i) holds.

Moreover $q(k) u(k-\rho) \leq b R(k)$ for $k \geq k_{2}$, and hence (ii) and (iii) follow. The proof when $u(k)$ is eventually negative is similar.

Example 3.12. For the generalized $\alpha$-difference equation

$$
\begin{gathered}
\Delta_{\alpha(\ell)}\left(\frac{1}{k} \Delta_{\alpha(\ell)}\left(\frac{1}{k^{2}}-\frac{1}{k(k-2 \ell)^{2}}\right)\right)=\frac{1}{\left((k+2 \ell)^{2}\right)_{\ell}^{(5)}(k+\ell)_{\ell}^{(2)}} \\
\left(k\left(k^{2}-k-2 \ell\right)\left(k^{2}-\ell^{2}\right)^{2}(k-2 \ell)^{2}-\alpha\left[(k-\ell)^{2}-(k+\ell)\right] k^{2}(2 k+\ell)\left(k^{2}-4 \ell^{2}\right)^{2}\right. \\
\left.+\alpha^{2}\left[(k-2 \ell)^{2}-k\right](k+2 \ell)^{2}\left(k^{2}-\ell^{2}\right)^{2}(k+\ell)\right)
\end{gathered}
$$

where $q(k)=\frac{-1}{k}, p(k)=\frac{1}{k}$ and the conditions of Theorem 3.2 holds. Infact $u(k)=\frac{1}{k^{2}}$ is one such solution.

We conclude with an oscillation theorem for (1) in the case $p(k) \equiv 1$ and $q(k) \equiv q>0$. Now, (1) takes the form

$$
\Delta_{\alpha(\ell)}^{2}(u(k)+q(k) u(k-\rho))+t(k) f(u(k-\tau))=0, k \in[0, \infty) .
$$

Theorem 3.13. Suppose that $t(k)$ is $k$-periodic and $f$ is nondecreasing and satisfies

$$
\begin{gathered}
f(u+v) \leq f(u)+f(v) \text { if } u, v>0, \\
f(u+v) \geq f(u)+f(v) \text { if } u, v<0, \\
f(c u) \leq c f(u) \text { if } c>0 \text { and } u>0 \\
f(c u) \geq c f(u) \text { if } c>0 \text { and } u<0 .
\end{gathered}
$$

Then every solution of (24) is oscillatory. 
Proof. Assume that (24) has a nonoscillatory solution and let $u(k)$ be eventually positive. Then $z(k)=u(k)+q u(k-\rho)>0$ eventually, say for $k \geq k_{1}$. From (24) we have $\Delta_{\alpha(\ell)}^{2} z(k) \leq 0$ for $k \geq k_{2} \geq k_{1}$. We claim that $\Delta_{\alpha(\ell)} z(k)>0$ for $k \geq k_{2}$. In fact, if for some $k_{3} \geq k_{2}, \Delta_{\alpha(\ell)} z\left(k_{3}\right) \leq 0$ then since $t(k) \neq 0$ there exists $k_{4}>k_{3}$ such that $\Delta_{\alpha(\ell)} z\left(k_{n}\right) \leq \Delta_{\alpha(\ell)} z\left(k_{4}\right)<0$ and by summation we see that $z(k) \rightarrow-\infty$ as $k \rightarrow \infty$. This contradicts the fact that $z(k)>0$ eventually.

Let $w(k)=z(k)+q z(k-\rho)$. Since from $(24)$ we have $\Delta_{\alpha(\ell)}^{2} z(k)=-t(k) f(u(k-$ $\tau))$, so, by the assumptions, we get

$$
\begin{aligned}
& \Delta_{\alpha(\ell)}^{2} w(k)+q \Delta_{\alpha(\ell)}^{2} w(k-\rho)+t(k) f(w(k-\tau))=-t(k) f(u(k-\tau)) \\
& -2 q t(n-\rho) f(u(k-\rho-\tau))-q^{2} t(k-2 \rho) f(u(k-2 \rho-\tau))+ \\
& t(k) f(u(k-\tau)+q u(k-\tau-\rho)+q(u(k-\tau-\rho)+q u(k-\tau-\rho))) \\
& \leq-t(k)\left(f(u(k-\tau))+2 q f(u(k-\tau-\rho))+q^{2} f(u(k-\tau-\rho))\right. \\
& +t(k)\left(f(u(k-\tau))+2 q f(u(k-\tau-\rho))+q^{2} f(u(k-\tau-2 \rho))\right)=0 .
\end{aligned}
$$

That is

$$
\Delta_{\alpha(\ell)}^{2} w(k)+q \Delta_{\alpha(\ell)}^{2} w(k-\rho)+t(k) f(w(k-\tau)) \leq 0,
$$

and observe that $w(k)>0$ and $\Delta_{\alpha(\ell)} w(k)>0$ for $k \geq k_{5}$, for some $k_{5} \geq k_{2}$. Therefore, $w(k-\tau)$ is increasing for $k \geq k_{6}$ for some $k_{6} \geq k_{5}$.

Applying Definition 2.1 and Lemma 2.2 to (25) from $k_{6}$ to $k-\ell$ we have

$$
\begin{gathered}
\Delta_{\alpha(\ell)} w(k)-\alpha^{\left\lceil\frac{k-k_{6}}{\ell}\right\rceil} \Delta_{\alpha(\ell)} w\left(k_{6}\right)+q \Delta_{\alpha(\ell)}^{2} w(k-\rho)-q \alpha{ }^{\left\lceil\frac{k-k_{6}}{\ell}\right\rceil} \\
\Delta_{\alpha(\ell)} w\left(k_{6}-\rho\right)+\sum_{r=0}^{\frac{k-k_{6}-j}{\ell}} \frac{t\left(k_{6}+j+r \ell\right) f\left(u\left(k_{6}+j+r \ell-\tau\right)\right)}{\alpha^{\left\lceil\frac{k_{6}+j-k+r \ell}{\ell}\right\rceil}} \leq 0 .
\end{gathered}
$$

By the monotonicity of $w(k)$ and $f$, it follows that

$$
\begin{gathered}
f\left(w\left(k_{6}-\tau\right)\right) \sum_{r=0}^{\frac{k-k_{6}-j}{\ell}} \frac{t\left(k_{6}+j+r \ell\right)}{\alpha^{\left\lceil\frac{k_{6}+j-k+r \ell}{\ell}\right\rceil}} \\
\leq \alpha{ }^{\left\lceil\frac{k-k_{6}}{\ell}\right\rceil} \Delta_{\alpha(\ell)} w\left(k_{6}\right)+p \alpha^{\left\lceil\frac{k-k_{6}}{\ell}\right\rceil} \Delta_{\alpha(\ell)} w\left(k_{6}-\rho\right),
\end{gathered}
$$

for $k \geq k_{6}$. Hence, there exists a constant $C$ such that

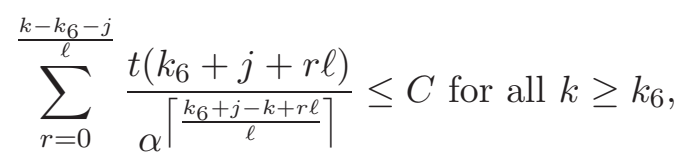


which contradicts (f). A similar argument can be used in the case of an eventually negative solution. This completes the proof.

Example 3.14. For the generalized $\alpha$-difference equation

$$
\Delta_{\alpha(\ell)}^{2}\left((-\alpha)^{\left\lceil\frac{k}{\ell}\right\rceil}+\frac{1}{k}(-\alpha)^{\left\lceil\frac{k-2 \ell}{\ell}\right\rceil}\right)=\frac{(-\alpha)^{\left\lceil\frac{k+\ell}{\ell}\right\rceil}}{(k+\ell) k}\left(2 k^{2}+2 k \ell+2 \alpha^{2} k+\alpha^{2} \ell\right)
$$

where $q(k)=\frac{1}{k}$, all the conditions of Theorem 3.13 hold and hence all solutions of (26) are oscillatory. Infact $u(k)=(-\alpha)^{\left\lceil\frac{k}{\ell}\right\rceil}$ is one such solution.

\section{Acknowledgments}

Research Supported by National Board for Higher Mathematics, Department of Atomic Energy, Government of India, Mumbai.

\section{References}

[1] R.P Agarwal, Difference Equations and Inequalities, Marcel Dekker, New York, 2000.

[2] Aleksandra Sternal and Blazej Szmanda, Asymptotic and Oscillatory Behaviour of Certain Difference Equations, Le Matematiche, LI(1996), Fasc.I, $77-86$.

[3] Jerzy Popenda and Blazej Szmanda, On the Oscillation of Solutions of Certain Difference Equations, Demonstratio Mathematica, XVII(1), (1984), $153-164$.

[4] Jerzy Popenda, Oscillation and Nonoscillation Theorems for Second-Order Difference Equations, Journal of Mathematical Analysis and Applications, 123(1), (1987), 34 - 38.

[5] M.Maria Susai Manuel, G.Britto Antony Xavier and E.Thandapani, Theory of Generalized Difference Operator and Its Applications, Far East Journal of Mathematical Sciences, 20(2), (2006), 163-171.

[6] M.Maria Susai Manuel and G.Britto Antony Xavier, Generalized Difference calculus of sequences of real and complex numbers, International Journal of Computational and Numerical Analysis and applications 6(4) (2004), 401-415. 
[7] M.Maria Susai Manuel, G.Britto Antony Xavier and E.Thandapani, Qualitative Properties of Solutions of Certain Class of Difference Equations, Far East Journal of Mathematical Sciences, 23(3) (2006), 295-304.

[8] M.Maria Susai Manuel, G.Britto Antony Xavier and E.Thandapani, Generalized Bernoulli Polynomials Through Weighted Pochhammer Symbols, Far East Journal of Applied Mathematics, 26(3) (2007), 321-333.

[9] M.Maria Susai Manuel and G.Britto Antony Xavier, Recessive, Dominant and Spiral Behaviours of Solutions of Certain Class of Generalized Difference Equations, International Journal of Differential Equations and Applications, 10(4) (2007), 423-433.

[10] M.Maria Susai Manuel, A.George Maria Selvam and G.Britto Antony Xavier, Regular Sink and Source in terms of Solutions of Certain Class of Generalized Difference Equations, Far East Journal of Applied Mathematics, 28(3) (2007), 441-454.

[11] M.Maria Susai Manuel, G.Britto Antony Xavier and V.Chandrasekar, Generalized Difference Operator of the Second Kind and Its Application to Number Theory, International Journal of Pure and Applied Mathematics, 47(1) (2008), 127-140.

[12] M.Maria Susai Manuel, G.Britto Antony Xavier, V.Chandrasekar and R.Pugalarasu, On Generalized Difference Operator of Third Kind and its Applications to Number Theory,International Journal of Pure and Applied Mathematics 53(1) (2009),69-82.

[13] M.Maria Susai Manuel, Adem Kilicman, G.Britto Antony Xavier, R.Pugalarasu and D.S.Dilip, $\ell_{2(\ell)}$ and $c_{0(\ell)}$ solutions of a second order generalized difference equation, Advances in Difference Equation, Submitted.

[14] M.Maria Susai Manuel, G.Britto Antony Xavier, D.S.Dilip and G.Dominic Babu, Oscillation, Nonoscillation and Growth of Solutions of Generalized Second Order Nonlinear $\alpha$-Difference Equations, Global Journal of Mathematical Sciences: Theory and Practical, 4(1), 2012, 211 - 225.

[15] M.Maria Susai Manuel, G.Britto Antony Xavier, D.S.Dilip and G.Dominic Babu, Oscillation and Nonoscillation for Certain Class of First and Second Order Generalized $\alpha$-Difference Equations, International Journal of Pure And Applied Mathematics, 78(3), 2012, 451 - 468. 
[16] M.Maria Susai Manuel, G.Britto Antony Xavier and D.S.Dilip, $\alpha$ Difference Operator And Its Application On Number Theory, J. of Mod. Meth. in Numer. Math., 3(2), (2012), 79 - 95.

[17] M.Maria Susai Manuel, G.Britto Antony Xavier, D.S.Dilip and G.Dominic Babu, Solutions of Certain Type of Second Order Generalized $\alpha$-Difference Equation in $L_{p}$ Space, International Journal of Advances In Pure And Applied Mathematics, Accepted.

[18] R.Pugalarasu,M.Maria Susai Manuel, V.Chandrasekar and G.Britto Antony Xavier, Theory of Generalized Difference operator of n-th kind and its applications in number theory (Part I), International Journal of Pure and Applied Mathematics, 64(1) (2010), 103-120.

[19] R.Pugalarasu,M.Maria Susai Manuel, V.Chandrasekar and G.Britto Antony Xavier, Theory of Generalized Difference operator of n-th kind and its applications in number theory (Part II), International Journal of Pure and Applied Mathematics, 64(1) (2010), 121-132.

[20] Ronald E.Mickens, Difference Equations, Van Nostrand Reinhold Company, New York, 1990.

[21] Saber N.Elaydi, An Introduction To Difference Equations, Second Edition, Springer, 1999.

[22] Walter G.Kelley, Allan C. Peterson, Difference Equations, An Introduction with Applications, Academic Press, inc 1991. 
